\title{
The effects of practicing speech accommodations to older adults
}

\author{
SUSAN KEMPER, MEGHAN OTHICK, HOPE GERHING, \\ JULIA GUBARCHUK, and CATHERINE BILLINGTON \\ University of Kansas
}

ADDRESS FOR CORRESPONDENCE

Susan Kemper, 426 Fraser, Psychology, University of Kansas, Lawrence, KS 66045

\begin{abstract}
This study evaluated the effects of practice with a referential communication task on the form and effectiveness of elderspeak, a speech register targeted at older listeners. The task required the listener to reproduce a route drawn on a map following the speaker's instructions. Young adults were given extended practice with this task to determine if they would modify their fluency, prosody, grammatical complexity, semantic content, or discourse style. The effectiveness of the young speakers' instructions was also evaluated in terms of how accurately their older partners could reproduce the routes and in terms of the older adults' evaluations of their own communicative competence. With practice, the young adults' instructions became shorter, simpler, slower, and more repetitious; these selective changes did not affect the older adults' accuracy, but did result in lower self-ratings of communicative competence by the older partners. In a second study, a new group of young adults was given extended practice with young adults as partners. The practice effects were limited to fluency (sentence length and speech rate) and had no effect on the young partners' accuracy or selfratings of communicative competence.
\end{abstract}

A special speech register sometimes termed "elderspeak" has been described as an accommodation to communicating with older adults. Elderspeak may be evoked by negative stereotypes of older adults as well as by their actual communicative needs; hence, elderspeak is addressed to healthy older adults as well as to those who are, or are presumed to be, cognitively impaired (Caporael, 1981; Caporael \& Culbertson, 1986; Caporael, Lukaszewski, \& Culbertson, 1983; Kemper, 1994; Ryan, Bourhis, \& Knops, 1991; Ryan, Giles, Bartolucci, \& Henwood, 1986). Elderspeak has been characterized as involving a simplified speech register with exaggerated pitch and intonation, simplified grammar, limited vocabulary, and slow rate of delivery. It appears to be a robust phenomenon that occurs in a wide range of settings involving older adults, such as craft classes, legal seminars, and congregate meals, as well as in nursing homes for demented and nondemented older adults (Kemper, 1994), although it is most often associated with nursing homes and other health care facilities (Ashbum \& Gordon, 1981; Gibb \& O’Brien, 1990; Gubrium, 1975; Lanceley, 1985; Ryan, Hummert, \& Boich, 1995). Many of the characteristics of elderspeak, such as its slow rate, exaggerated prosody, and simplified syntax and vocabulary, resem- 
Kemper et al.: Practicing speech accommodations to older adults

ble the characteristics of other speech registers, such as those directed at young children, foreigners, and household pets (Warren \& McCloskey, 1997).

Kemper and her collaborators (Kemper, Othick, Warren, Gubarchuk, \& Gerhing, 1996; Kemper, Vandeputte, Rice, Cheung, \& Gubarchuk, 1995) demonstrated that young adults spontaneously adopt a simplified speech register when addressing older listeners during a referential communication task. The referential communication task used in these studies required the listener to reproduce a route drawn on a map following directions given by the speaker. Dyads of young-young, older-older, and young-older adults alternated as speakers and listeners. Older adults showed little variation in their speech style, regardless of whether they were addressing young or older listeners. Young adults, however; adopted a simplified speech style when instructing older listeners; the simplified style not only provided more information in terms of words, utterances, instructions, and location checks on the listener's progress, but also "packaged" this information differently. The young adults paused more often, used shorter sentences, used few complex syntactic constructions, and reduced the informational content of individual utterances by lowering propositional density (Kintsch \& Keenan, 1973). These speech adjustments appeared to benefit the older listeners, who were able to reproduce the maps more accurately than when they were paired with older speakers. Older adults' speech to young adults versus other older adults did not vary in fluency, grammatical complexity, semantic content, or discourse style. The older adults may have used a consistent speech register for a variety of reasons: they may have lacked the processing resources necessary to produce complex grammatical constructions and constructions high in propositional density (Kemper, 1992); they may not have been sensitive to the same situational or interpersonal cues that elicit code-switching by young adults; or they may have been unwilling to adopt a form of babytalk when addressing peers.

The young adults' use of elderspeak may have been triggered by beliefs about the communicative competence of older adults or by actual communicative problems of their older partners, as signaled by their older partners' behavior during the referential communication task. Older listeners frequently interrupted speakers to repeat the speakers' instructions, request clarification, or express confusion or difficulty with the task. These interruptions may have cued the young adults to simplify their speech. In order to investigate this possibility, Kemper et al. (1996) replicated the study with one major change: listeners were prohibited from interrupting the speaker to request clarification or express confusion. In the second study, young adults again spontaneously adopted a simplified speech register when addressing older listeners during the referential communication task. This speech register resembled other forms of elderspeak in that it was marked by reductions in speaking rate, sentence length, the use of complex syntactic constructions, and propositional density, as well as by increases in words, utterances, instructions, and repetitions. The young adults' use of elderspeak in the second study was not cued by the actual behavior of the older listeners during the task since the listeners were prohibited from interrupting the speakers. Rather, the young speakers were drawing upon a set of stereotypes of the communicative problems of older adults in order to modify their speech. 
In both Kemper et al. (1995) and Kemper et al. (1996), the young adults' use of elderspeak improved the performance of the older adults on the referential communication task; older listeners performed more accurately when paired with young speakers than when paired with other older adults. The young speakers' decrease in sentence length, grammatical complexity, and propositional density and their increase in words, utterances, instructions, repetitions, and location checks were associated with lower error scores by the older listeners. Working memory limitations appear to impair the older adults' performance on the referential communication tasks; it is likely that the young adults' speech accommodations reduced working memory demands because they provided more information and packaged that information into shorter and simpler utterances (Kemper, 1992; Kemper, Anagnopoulos, Lyons, \& Heberlein, 1994).

It is important to note that in these studies the young adults' use of elderspeak was not without cost. Although older adults did better on the referential communication task when paired with young partners, they reported more expressive and receptive communication problems. Their self-reported communication problems with young partners were associated with shorter sentences, slower speaking rate, higher pitch, greater pitch range, and more speaker repetitions. These stylistic factors appear to trigger older adults' perceptions of themselves as communicatively impaired, leading to increased self-reports of expressive and receptive problems. The older adults' self-reported expressive and receptive problems were not associated with the young adults' grammatical complexity or semantic content and repetitions.

Similar consequences of the use of elderspeak have been noted by other researchers. Harwood, Giles, and Ryan (1995) argued that the use of elderspeak, as well as other age-based behavioral modifications, contributes to the development of an "old" identity by reinforcing negative stereotypes of older adults and lowering older adults' self-esteem. Following Ryan, Giles, Bartolucci, and Henwood (1986), they suggested that a downward spiral can result such that elderspeak contributes to the social isolation and cognitive decline of older adults, triggering further speech simplifications. Other observational 'studies, as well as simulation studies using scripted interactions, have noted that elderspeak conveys a sense of disrespect toward its recipients, limits their conversational interactions, and implies that they are cognitively impaired (Edwards \& Noller, 1993; Gubrium, 1975; Ryan et al., 1991; Ryan, Hamilton, \& Kwong See, 1994; Ryan, MacLean, \& Orange, 1994).

O'Connor and Rigby (1996) examined how community-dwelling older adults and nursing home residents perceive elderspeak versus more neutral speech. Written scripts were rated by the participants on a number of adjectives such as "warm," "shrill," and "superior." The babytalk or elderspeak script was rated lower in warmth and higher in superiority than the neutral script. Nursing home residents perceived less superiority in elderspeak than did the community-dwelling older adults, and the oldest participants in the study perceived less superiority in elderspeak than did the younger participants. O'Connor and Rigby, following Ryan et al. (1991), suggested that older adults, especially those in nursing homes, adapt to situational demands by becoming more accepting of elderspeak. Ratings of self-esteem and the frequency of receiving babytalk were also collected by O'Connor and Rigby (1996). Lower self-esteem was reported 
Kemper et al.: Practicing speech accommodations to older adults

by women who frequently received babytalk and who rated it negatively in warmth; higher self-esteem was reported by men who rarely received babytalk and who rated it highly in superiority.

Although the psycholinguistic characteristics and sociolinguistic consequences of elderspeak have begun to be investigated, this speech register is still poorly understood. To date, research has not addressed such questions as: How effective is elderspeak with regard to enhancing the performance of older adults? How much experience with older adults is required in order to master this speech register? How do task demands and contextual factors affect the form or function of elderspeak? The available research base is far from adequate to answer these questions. The present study was undertaken to begin to address these problems by examining the effects of extended practice on the use of elderspeak by young adults during a referential communication task with older adults. Young adults participated in five sessions, each time paired with different older adults. Their speech to their older partners from the first session was compared with that from the fifth session to determine how elderspeak might be affected by practice and whether such practice effects might affect older adults' self-ratings of communicative competence. Practice was expected to result in a modified form of elderspeak that would improve the older adults' accuracy on the task and, perhaps, increase their self-ratings of communicative competence. The modified form of elderspeak was expected to convey more semantic information via shorter and less complex utterances.

\section{EXPERIMENT 1}

\section{Method}

Subjects. The participants in this study were 10 young adults and 50 older adults. The young adults were between 18 and 25 years of age $(M=22.8$ years); $80 \%$ were women. The older adults were between 60 and 84 years of age $(M=$ 74.1 years); $64 \%$ were women. The two groups did not differ in the number of years of education they had completed $(M=15.2$ years), nor did they differ in their performance on the Shipley (1940) vocabulary test ( $M=33$ of 40 correct).

The young adults were recruited by posted signs and other announcements; they were paid $\$ 10$ for each test session plus a bonus of $\$ 50$ if they completed all five sessions. The bonus was paid to ensure that the young adults would complete all five sessions. The older adults were recruited from a registry of previous research participants; all were living at home alone or with family. The older adults were paid $\$ 20$ for each test session, which included compensation for their travel to campus to participate in this research.

Task and materials. Each young adult participated in five sessions, each time paired with a different older adult as a partner. The sessions were scheduled on successive days. All sessions followed the same format.

At each session, the participants were introduced to each other, and basic demographic information such as their ages, occupations, and educational history was collected. The two participants then engaged in an 8-minute "get ac- 
quainted" conversation. They were instructed to discuss the events of the day. These conversations were audiorecorded. Then they were introduced to the referential communication task (Molfese, Hoffman, \& Yuen, 1981-1982; Rubin \& Brown, 1975; Warren \& McCloskey, 1997). A folding screen was placed between the two subjects so that they could hear but not see each other. The experimenter first demonstrated the referential communication task, and then one round of practice was held so that each subject could practice the task, once as a speaker and once as a listener. Following this practice, the young adult was designated as the first speaker and was given a map of a hypothetical city with labeled streets and landmarks. A route was drawn on the city map with a beginning location and a final terminus indicated. The older adult was designated as the listener and was given a similar map without the indicated route. The speaker was instructed to "describe the route so that the listener can trace it on her [or his] map." No other instructions were given. When the speaker had finished or 5 minutes had elapsed, the subjects exchanged roles using a new map with a different route. Each dyad completed four rounds of participation so that each subject served as the speaker for four different maps and the listener for four different maps. Both participants then filled out a modified version of the Language in Adulthood Questionnaire (LAQ) (Ryan, Kwong See, Meneer, \& Trovato, 1992) to assess their own receptive and expressive communicative competence.

The LAQ (Ryan et al., 1992) was modified slightly so that it referred to expressive or receptive problems with this task. There were nine questions about receptive problems such as "My partner spoke too fast" or "I often had to ask my partner to repeat." There were eight questions about expressive problems such as "I lost track of what I was trying to say" and "I had trouble telling the route." Each was accompanied by a 7-point rating scale, ranging from (1) strong disagreement to (7) strong agreement. Higher ratings, therefore, indicate more expressive or receptive problems and hence a lower level of communicative competence.

Analysis. This research used the language sample analyses of Kemper et al. (1995) and Kemper et al. (1996) to assess the nature of the speech addressed to older adults and the effects of extended practice on the form and effectiveness of such elderspeak. The young adults' instructions at sessions 1 and 5 were analyzed as were their partners' responses on these days. The language samples collected on sessions 2, 3, and 4 were not analyzed. The older adults' instructions at sessions 1 and 5 were also compared, as were their young partners' responses. This approach involved the systematic analysis of the speakers' directions and listeners' responses for the map problems (Kemper et al., 1995; Kemper et al., 1996). To ensure an adequate sample, the speakers' directions for all four maps used in each session were combined for the analysis, as were the listeners' responses.

Verbal fluency was assessed by several measures. First, mean length of utterance (MLU) in words was used as a general measure of fluency. The incidence of sentence fragments and the total number of words and utterances per map were used as additional measures of fluency. Sentences were required to have a 
Kemper et al.: Practicing speech accommodations to older adults

main verb, usually expressed as an imperative such as "go" or "turn." Fragments typically lacked a verb. Many utterances lacked a verb, but provided a continuation or extension of a previous utterance after a pause; for this analysis, these utterances were coded as fragments. The final measure of fluency was the speaker's speaking rate in words per minute (wpm). This rate was sampled at five points by timing the duration of three to five contiguous utterances by the speaker during each task and computing an average speaking rate for each speaker in words per minute. The contiguous utterances were permitted to contain filled or unfilled pauses as well as repetitions of words or phrases. The utterances were timed by digitizing the segments and measuring the duration of the segments on a spectrograph.

Prosody for each speaker was assessed by a spectrographic analysis of selected segments uttered by each speaker during each task. Matched segments were selected from the speakers' directions to older versus younger listeners such that they were identical lexically and occurred in highly similar syntactic configurations. For example, the phrase "go one block north" was extracted as part of the utterance "And you go one block north," addressed to an older listener, and as part of the utterance "Then you tum left and go one block north," addressed to a younger listener by the same speaker. Segments were required to contain fluent, continuous speech without discernible pauses or repetitions. Five such pairs of matched segments were selected for analysis from each session by each speaker. Seven measures were obtained from each segment using the Computerized Speech Research Environment (AVAAZ Innovations, 1995) software. The measures from each segment were averaged for the five segments selected from each speaker for each target: the highest pitch and the lowest pitch were extracted by automatic pitch analysis, the pitch range was computed as the difference between the highest and lowest pitches, the average pitch was computed by sampling the pitch peaks of three to five vowels occurring in the segment, and the average values for the F1, F2, and F3 formats for these vowels was determined by automatic format frequency extraction using linear predictive analysis procedures. Finally, the duration of each segment was determined by spectrographic analysis from the onset to the offset of the target segment. Amplitude (or loudness). could not be reliably measured given the recording situation.

Grammatical complexity was assessed by analyzing the grammatical structure of each speakers' utterances. The analytic procedures of Kemper et al. (1995) and Kemper et al. (1996) were adopted. Measures of syntactic complexity included one composite measure, mean number of clauses per utterance (MCU), and two measures of clause composition: the incidence of left-branching and right-branching clauses per utterances. Left-branching clauses included sentence-initial subordinate clauses, as well as relative clauses modifying the sentence subject and that- and wh-clauses used as sentence subjects. Rightbranching clauses included sentence-final subordinate clauses, as well as relative clauses modifying the sentence predicate, infinitive complements, and that-and wh-complements.

Four measures of the semantic content, or the amount of information con- 
veyed by the speaker, were computed. Type/token ratios of the number of different words to the total number of words were computed as a measure of lexical diversity. Propositional density (Kintsch \& Keenan, 1973), or the number of propositions per 100 words, was determined as a measure of information content. (A proposition is a basic idea unit, typically a verb and its noun phrase arguments, an adjective or adverb, or a prepositional phrase.) Causal and temporal relations among propositions were also scored as propositions. The number of instructions used to describe each map was tallied. An instruction was required to have two components: a verb such as "go" or "turm" and a direction such as "Go to the top row" or "go diagonally down to the left." Instructions optionally conveyed distances, as in "Go north three blocks." A measure of redundancy was also computed by tallying the number of instructions that the speaker repeated, as in "Go north three blocks ... north three blocks ... then turn left."

The structure of these interactions was analyzed with regard to the speakers' interaction style and the listeners' style. The speakers' style was analyzed with regard to the number of speaker tums per map and the number of speaker utterances per turn to measure how long each speaker was able to sustain or dominate the interaction. A tum was defined as a sustained sequence of utterances not interrupted by remarks from the listener. Backchannel affirmatives were not considered as interrupting the speakers' turns. A third measure of the speakers' style was the number of location checks provided by the speaker as in statements such as "We are now at the comer of Oak Street and First Avenue" or queries such as "Are you at the post office now?"

The listeners' style was also analyzed. The number of instructions repeated by the listener was tallied. The number of backchannel affirmatives was tallied per map. Backchannel affirmatives were used to mark comprehension or agreement with the speakers' instructions and typically coincided or overlapped with the speakers' utterances. Affirmative or negative answers to questions were not scored as backchannels, but were treated as separate utterances. The listeners' requests for clarification, as well as their expressions of confusion per map, were also scored as listener style measures. An example of a clarification request would be "Do you mean go west?" Expressions of confusion included remarks such as "I'm so lost" and "Wait a moment until I find where I am." Table I illustrates these analyses of the speakers' fluency, grammatical complexity, semantic content, and speakers' and listeners' discourse style.

Finally, the listener's maps were scored for accuracy. Two measures were computed. First, it was determined whether the listener's maps exactly duplicated the speaker's; all intermediate paths, locations, and turnings were required to be correct, as well as the final terminus. Since there were many incorrect solutions as well as incomplete maps due to the 5-minute time limit, a second accuracy measure was also computed for maps that were incomplete or incorrect. The listener's map was superimposed over the speaker's, and all errors were measured in city block units. City block units were defined by map dịrections and corresponded to the minimum number of line segments (corresponding to street blocks) by which the listener's map differed from the speaker's. 
Kemper et al.: Practicing speech accommodations to older adults

Table 1. Extract from one referential communication session

S: Start [main clause] over on the right-hand side at the department store. [instruction]

S: Go [main clause] to Third Street [instruction] and tum [main clause] left. [instruction]

L: Yeah ${ }^{\wedge}$ [backchannel]

S: Now go [main clause] two blocks until you pass [right-branching clause] the bank ${ }^{\wedge}$ [instruction]

S: Are [main clause] you at the bank? [location check]

L: Yes.

L: I'm at the bank on Third Street.

S: $\quad \mathrm{OK}^{\wedge}$

S: Now go [main clause] north one block to Fourth Street. [instruction]

L: Yeah ${ }^{\wedge}$ [backchannel]

S: And go [main clause] one block. [instruction]

S: Before you get to city hall [left-branching clause] ${ }^{\wedge}$

S: Turn [main clause] [instruction] and go [main clause] north all the way to Eighth Street. [instruction]

S: Are [main clause] you with me? [location check]

L: Am I [main clause] on Eighth Street? [request for clarification]

S: Once you get [left-branching clause] to Eighth Street, tum [main clause] right [instruction]

Note: S, speaker (young adult); L, listener (older adult); ${ }^{\wedge}$, end of fragment; [ ], codes for the measures of grammatical complexity, semantic content, speaker style, and listener style.

Reliability. The reliability of the analysis was assessed in three ways. First, all transcripts of the interactions were prepared by one trained coder and verified by a second coder. The two coders also coded five interactions independently with regard to the determination of sentences and fragments and the measures of grammatical complexity; agreement was high (Cronbach's alpha $>.90$ for each measure). The analysis of speaker and listener discourse styles was coded from the prepared transcripts by a third coder, and a fourth coder independently coded five interactions for these measures (for these measures, Cronbach's alpha $>$.90). These two coders also independently scored five listeners' maps for accuracy; they agreed on all five maps with regard to solution accuracy (Cronbach's alpha $=.90$ for the error score). A fifth coder computed the speaking rates, and these rates were also computed by the fourth coder for five speakers. The two coders differed by no more than five words per minute for any of the five speakers (Cronbach's alpha $=.90$ ). Prosody was determined by one analyst as well as by a second analyst for five speakers with an average accuracy of $+25 \mathrm{~Hz}$ for all pitch and format values and $+15 \mathrm{msec}$ for the segment durations (Cronbach's alpha $>.80$, for all measures). Word and utterance counts, type/ token ratios, and mean lengths of utterance were computed by the SALT (Chapman \& Miller, 1984) computer program, which was used to facilitate all analyses. 
Kemper et al.: Practicing speech accommodations to older adults

Table 2. Means and standard deviations (in parentheses) for the language sample measures of the young speakers at sessions $I$ and 5

\begin{tabular}{|c|c|c|c|c|c|}
\hline \multirow[b]{2}{*}{ Fluency } & \multicolumn{2}{|c|}{ Session 1} & \multicolumn{2}{|c|}{ Session 2} & \multirow[t]{2}{*}{$F(1,9)=$} \\
\hline & & & & & \\
\hline MLU & 5.9 & $(.8)$ & 5.2 & $(.5)$ & $12.67 * *$ \\
\hline Total words & 862 & (81) & 843 & (75) & $<1.0$ \\
\hline Total utterances & 157 & (35) & 172 & (21) & $<1.0$ \\
\hline Percent fragments & 45 & (15) & 62 & (12) & $13.22 * *$ \\
\hline Rate in words/minute & 92 & $(9)$ & 77 & (6) & $12.65^{* *}$ \\
\hline \multicolumn{6}{|l|}{ Prosody } \\
\hline Duration & 691 & $(220)$ & $676)$ & (155) & $<1.0$ \\
\hline Pitch range & 1429 & (158) & 1472 & (159) & $<1.0$ \\
\hline Average pitch & 222 & $(36)$ & 213 & (29) & $<1.0$ \\
\hline $\mathrm{Fl}$ & 644 & (78) & 658 & (79) & $<1.0$ \\
\hline F2 & 1306 & (236) & 1407 & (239) & $<1.0$ \\
\hline F3 & 2243 & (397) & 2269 & (329) & $<1.0$ \\
\hline \multicolumn{6}{|l|}{ Grammatical complexity } \\
\hline $\mathrm{MCU}$ & 1.6 & $(.1)$ & .6 & $(.1)$ & $13.99 * *$ \\
\hline Percent left-branching & 3 & (1) & 1 & (1) & $13.66 * *$ \\
\hline Percent right-branching & 25 & (2) & 13 & (4) & $11.21^{* *}$ \\
\hline Percent main clauses & 72 & (3) & 86 & (4) & $9.87^{*}$ \\
\hline \multicolumn{6}{|l|}{ Semantic content } \\
\hline Type/token ratio & 28 & (10) & 30 & (9) & $<1.0$ \\
\hline Propositions/100 words & 36 & (9) & 47 & (11) & $6.65^{*}$ \\
\hline Total instructions & 14 & (9) & 17 & (10) & $11.38 * *$ \\
\hline Repetitions/map & 3 & (4) & 7 & $(1)$ & $8.54^{*}$ \\
\hline \multicolumn{6}{|l|}{ Speaker style } \\
\hline Total turns & 3 & (4) & 2 & (1) & $<1.0$ \\
\hline Instructions/tum & 5 & (9) & 6 & (6) & $<1.0$ \\
\hline Location checks & 3 & (2) & 2 & (2) & $<1.0$ \\
\hline \multicolumn{6}{|l|}{ Listener style } \\
\hline Repetitions/map & 1 & (1) & 1 & (2) & $<1.0$ \\
\hline Backchannel affirmatives & 11.3 & (8) & 7.92 & $(6)$ & 1.01 \\
\hline Requests for clarification & 3.8 & (4) & 1.8 & (2) & 2.38 \\
\hline Expressions of confusion & .5 & (1) & .3 & (1) & $<1.0$ \\
\hline \multicolumn{6}{|l|}{ Accuracy } \\
\hline Percent correct maps & 85 & $(21)$ & 85 & (29) & $<1.0$ \\
\hline Errors in city blocks & .2 & $(.3)$ & .5 & $(1.2)$ & $<1.0$ \\
\hline
\end{tabular}

${ }^{*} p<.05 ;{ }^{* *} p<.01$

\section{Results}

YOUNG ADULTS' SPEECH. The primary analysis was performed on the language samples from the young adults. Means for each linguistic measure, as well as the mean accuracy scores, are presented in Table 2.

A series of multivariate analyses of variance were performed on the measures of fluency, prosody, grammatical complexity, semantic content, speaker style, 
Kemper et al.: Practicing speech accommodations to older adults

listener style, and accuracy with session (1 vs. 5) as a within-subject factor. The multivariate effects for fluency, grammatical complexity, and semantic content were significant $(p<.01)$; the multivariate effects for prosody, speaker style, listener style, and accuracy were not significant. All univariate effects are given in Table 2.

Fluency. The multivariate test was significant $(p<.01)$, as were the univariate tests for three of the five measures. The total number of words and the total number of utterances used by the young adults to describe the routes did not differ from session 1 to session 5; however, they used shorter utterances and more sentence fragments after extended practice with older adults. In addition, their speech rate was somewhat slower, measured in terms of words per minute, in session 5 than in session 1.

Prosody. The young adults did not vary their prosody from session 1 to session 5. The multivariate test was nonsignificant, and the univariate tests for all six measures (segment duration, pitch range, average pitch, and F1, F2, F3) were nonsignificant. The finding that the prosody measure of segment duration did not differ across the two sessions in conjunction with the finding that the fluency measure of speech rate in words per minute did decline suggest that the decline in speech rate was achieved by pausing longer between words or phrases rather than by prolonging individual words or phrases.

Grammatical complexity. The multivariate effect for grammatical complexity was significant $(p<.01)$, as were all five univariate effects. The young adults simplified their speech after extended practice with the referential communication task involving older adults. By session 5, they were using fewer clauses per utterance, lowering MCU, and had reduced the incidence of left- and rightbranching clauses. Consequently, the percentage of main clauses increased from session 1 to session 5 .

Semantic content. The multivariate effect for semantic content was significant $(p<.05)$, as were three of the four univariate effects. Only type/token ratios failed to show an effect of extended practice with the referential communication task. The young adults produced fewer propositions per 100 words, gave more instructions per map, and repeated those instructions more often during session 5 than during session 1. Hence, the overall semantic content of their route descriptions increased with practice.

Speaker and listener style. None of the discourse style measures showed an effect of practice. The multivariate effects for the speaker style and listener style measures were both nonsignificant $(p>.50)$; none of the univariate effects was significant. Speakers used as many turns per map, instructions per tum, and location checks during session 1 as during session 5; listeners repeated instructions and produced backchannel affirmatives, requests for clarification, and expressions of confusion as often during session 1 as during session 5. 
Kemper et al.: Practicing speech accommodations to older adults

Accuracy. The older adults' accuracy was unchanged from session 1 to session 5. The multivariate effects were nonsignificant, as were both univariate effects.

OLDER ADULTS' SPEECH. The language sample analysis was repeated to compare the older speakers from session 1 with those from session 5 . None of the multivariate effects and none of the univariate effects (fluency, prosody, grammatical complexity, semantic content, speaker style, and listener style measures) were significant (all $p>.10$ ). This indicates that the older speakers at the first and fifth sessions did not differ with regard to speech style.

Post-hoc analyses confirmed prior findings (Kemper et al., 1995; Kemper et al., 1996) with regard to the relative fluency, complexity, content, and style of young versus older speakers during session 1. Young speakers differed from older speakers on most measures at $p<.05$. With regard to the fluency measures, young speakers had higher mean length of utterance ( 5.9 vs. 5.2), used fewer words (862 vs. 638) and more utterances (157 vs. 107) per map, and spoke more rapidly than older speakers $(92 \mathrm{vs.} 80 \mathrm{wpm})$. Their prosody also differed from that of older speakers: phrase durations were shorter (691 vs. 822 $\mathrm{msec}$ ), pitch range was greater (1429 vs. $1065 \mathrm{~Hz}$ ), average pitch was higher (1222 vs. $1023 \mathrm{~Hz}$ ), and all three formats were higher $(644,1306$, and $2243 \mathrm{~Hz}$ vs. 442,1125 , and $1981 \mathrm{~Hz}$, respectively, for F1, F2, F3). Young speakers also used more complex utterances, with higher MCUs (1.6 vs. 1.3) and more rightand left-branching constructions (3\% and $25 \%$ vs. $1 \%$ and $3 \%$, respectively, for right- and left-branching constructions). Young speakers also provided more semantic content; their propositional density (or propositions per 100 words) was higher (36 vs. 26). Young adults produced more instructions per map (14/ map vs. 10/map), more repetitions per instruction (3.0/instruction vs. $0.2 /$ instruction), and more location checks per map (3.0/map vs. 1.5/map). Finally, maps drawn in response to young speakers were more accurate than those drawn in response to older speakers (85\% correct with 0.2 city block errors vs. $71 \%$ correct with 10.9 city block errors, respectively).

Communicative competence ratings. The responses to the nine questions about receptive problems and the eight questions about expressive problems on the modified LAQ were averaged for the younger and older adults for each session. Analysis of variance was used to analyze the LAQ ratings with session and respondent (young vs. older adults) as factors for both the expressive and receptive items. The main effect of respondent was significant, $F(1,9)=7.83$, $p<.05$. Young subjects strongly disagreed with all items, indicating that they experienced few receptive or expressive problems. Older adults rated themselves higher than the young adults with regard to both expressive and receptive problems, indicating that they perceived their communicative competence to be somewhat lower than did the young adults. The session-by-respondent interaction was also significant, $F(1,9)=5.78, p<.05$.

As indicated in Table 3, the young adults' ratings of their receptive and expressive problems did not differ from session 1 to session 5, whereas the older adults' ratings increased. That is, older adults who participated in session 5 rated themselves as communicatively less competent than those who participated in 
Kemper et al.: Practicing speech accommodations to older adults

Table 3. Younger and older adults' ratings of their own expressive and receptive communicative competence after sessions 1 and 5

\begin{tabular}{lccccc}
\hline \hline & \multicolumn{2}{c}{ Session 1 } & & \multicolumn{2}{c}{ Session 5 } \\
\cline { 2 - 3 } \cline { 5 - 6 } & Expressive & Receptive & & Expressive & Receptive \\
\hline Young adults & $1.8(.5)$ & $1.8(.6)$ & & $1.9(.4)$ & $1.7(.5)$ \\
Older adults & $2.6(.7)$ & $3.1(.6)$ & & $3.6(.7)$ & $3.9(.8)$ \\
\hline \hline
\end{tabular}

session 1. Although the older adults who participated in session 5 were as accurate in their map completions and used the same speech style as those who participated in session 1, the older participants in session 5 reported more expressive and receptive communication problems than did those who participated in session 1.

To investigate this issue further, the older adults' LAQ ratings for expressive and receptive problems were averaged and then correlated with the speech characteristics of their young partners. The data from sessions 1 and 5 were combined for this analysis. As shown in Table 4, LAQ ratings for communicative problems were significantly correlated with the young adults' mean length of utterance, pitch range, highest pitch (F3), and repetitions. These results confirm the findings from Kemper et al. (1996). The older adults reported more expressive and receptive problems when paired with young partners who used short sentences, high and exaggerated pitch, and many repetitions. The young adults' shift from session 1 to session 5 with regard to sentence length, speech rate, and repetitions appears to have lowered the older adults' self-assessed communicative competence.

\section{Discussion}

Young adults adopt a simplified speech style when instructing older adults during a referential communication task, and they modify this speech style as a result of extensive practice with the task. Elderspeak - at least the version used in this task - provides more information and presents that information more efficiently than speech addressed to young partners. Young speakers use more words, more utterances, more instructions, and more location checks when addressing older partners, and they speak more slowly, use shorter sentences, avoid grammatical complexities, and reduce propositional density (Kemper et al., 1995; Kemper et al., 1996). With practice, elderspeak acquires more distinctive characteristics: it gets even shorter, slower, and less complex and becomes more repetitious. Whereas the form of elderspeak spontaneously adopted by young adults appears to enhance the performance of older adults when they first encounter the referential communication task, the version that results from extensive practice with the task is not necessarily any better. Contrary to the predictions, the modified form of elderspeak used after four days of practice with the referential communication tasks did not enhance the older adults' accuracy. More importantly, although the streamlined form of elderspeak used after 
Kemper et al:: Practicing speech accommodations to older adults

Table 4. Correlations between the older adults' $L A Q$ ratings and characteristics of their young partners' speech

\begin{tabular}{lc}
\hline \hline & $r(18)$ \\
\hline Fluency & \\
MLU & $-.48^{*}$ \\
Total words & -.11 \\
Total utterances & +.04 \\
Percent fragments & +.08 \\
Rate in words/minute & -.42 \\
Prosody & \\
Duration & +.35 \\
Pitch range & $+.44^{*}$ \\
Average pitch & +.32 \\
F1 & +.11 \\
F2 & +.28 \\
F3 & $+.52^{*}$ \\
Grammatical complexity & \\
MCU & +.10 \\
Percent left-branching & +.04 \\
Percent right-branching & -.03 \\
Percent main clauses & -.10 \\
Semantic content & \\
Type/token ratio & -.14 \\
Propositions/lo0 words & +.05 \\
Total instructions & +.18 \\
Repetitions/map & $+.45^{*}$ \\
Speaker style & -.04 \\
Total turns & -.03 \\
Instructions/turn & +.21 \\
Location checks & \\
\hline \hline
\end{tabular}

Note: Data from sessions 1 and 5 are combined. ${ }^{*} p<.05$.

extensive practice did not affect the older adults' performance, it did lower their self-assessments of their own communicative competence. Older listeners reported experiencing more expressive and receptive problems when they were paired with young speakers who used exaggerated prosody and short, simple, repetitious sentences - the version of elderspeak used by well-practiced young speakers.

\section{EXPERIMENT 2}

In order to determine whether the practice effects for the young speakers resulted from their experiences in attempting referential communication with older adults or from their experience with the task itself, the study was repeated using young adults as listeners as well as speakers. 
Kemper et al.: Practicing speech accommodations to older adults

\section{Method}

Subjects. Participating in this study were 60 young adults. They were between the ages of 18 and 26 years of age ( $M=23.4$ years); 28 were women. Ten were recruited to participate in series of sessions over five consecutive days; the remaining 50 were recruited to participate in a single session. The two groups did not differ in age, years of education completed $(M=14.8$ years), or performance on the Shipley (1940) vocabulary test ( $M=34$ of 40 correct), nor did the subgroups of young adults participating during session 1 differ from those who participated during session 5 with regard to age, education, or vocabulary. All were paid $\$ 10$ per session for their participation. Those who participated in multiple sessions were paid a bonus of $\$ 50$ if they completed all five sessions.

Task, materials, analysis, and reliability. Ten of the young adults participated in five sessions, each time paired with a different young adult partner. The sessions followed the same format used in Experiment 1. The analysis followed the same procedures as those used in Experiment 1; the speech of the young adults during session 1 was compared with their speech during session 5 with regard to verbal fluency, prosody, grammatical complexity, semantic content, speaker and listener style, and accuracy. Reliability was assessed using similar procedures to those used in Experiment I (Cronbach alpha $>.90$ for all comparisons).

\section{Results}

A series of multivariate analyses of variance were performed on the measures of fluency, prosody, grammatical complexity, semantic content, speaker style, listener style, and accuracy with session ( 1 vs. 5) as a within-subject factor. With the exception of the multivariate effect for fluency, none of the multivariate effects were significant, although the statistical power to detect differences in prosody, complexity, content, style, or accuracy may have been limited by the small sample size.

In contrast to Experiment 1, the young adults became faster in terms of words per minute from session 1 to session 5 when paired with young adults as listeners $(M=95 \mathrm{wpm}$ vs. $115 \mathrm{wpm}, p<.05$, in sessions 1 and 5 , respectively); they used fewer words $(M=851$ vs. $754, p<.05)$ and fewer utterances (142 vs. 124, $p<.05$ ). As in Experiment 1, they also used shorter sentences (MLU $=5.8$ vs. $5.2, p<.05$, in sessions 1 and 5 , respectively) and more fragments (42\% vs. $68 \%, p<.05)$. These practice effects on the young adults' fluency did not affect the performance of their young adult partners. The young adults were extremely accurate at both sessions 1 and $5(M=97 \%$ correct).

The LAQ ratings were averaged for the nine questions about receptive competence and the eight questions about expressive competence and were analyzed using an analysis of variance with session (1 vs. 5) and respondent (practiced group vs. unpracticed group) as factors. There were no significant effects for either receptive or expressive items, although the small sample size may have limited the statistical power of the test to detect small differences. Young adults 
Kemper et al.: Practicing speech accommodations to older adults

rated their communicative competence as quite high at both sessions $(M=1.6$ and 1.7 for expressive items and 1.7 and 1.5 for receptive items, for sessions 1 and 5 , respectively).

The language samples collected during session 1 from the young adults who participated in all five sessions were compared with those from the young adults who participated only in session 1 . All multivariate tests were nonsignificant, indicating that the two groups did not differ initially in their fluency, prosody, grammatical complexity, semantic content, speaking style, listening style, or accuracy.

\section{Discussion}

Young adults who are given extensive practice with a referential communication task with other young adults as partners show little in the way of systematic changes to their speech. They do become somewhat faster and use shorter sentences and fewer words and utterances. These practice effects on speech fluency do not appear to impact their partners' accuracy or ratings of communicative competence. When partnered with other young adults, familiarity with a routine, nonchallenging task results in a more telegraphic speech style.

\section{CONCLUSION}

The results of Experiments 1 and 2 provide empirical confirmation of the hypothesis of Ryan et al. (1986) regarding the "communicative predicament of aging." Ryan et al. suggested that young adults' negative stereotypes of older adults lead to the use of simplified, controlling, and patronizing speech. As a result, older adults have more restricted communicative interactions, which may maintain and reinforce their own negative stereotypes of aging and trigger social withdrawal; hence, restrictive communicative interactions may further the social, physical, and cognitive decline of older adults. The "communicative predicament of aging," then, is that elderspeak can lead to a negative spiral of perceived and actual communicative impairments, yet the failure to use appropriate speech accommodations for older adults may also lead to social isolation and cognitive decline. The present findings, together with those of Kemper et al. (1995) and Kemper et al. (1996), confirm part of this downward spiral: young adults spontaneously adopt a simplified speech register when giving instructions to older adults, and older adults report more communicative problems, in accordance with negative stereotypes of aging, when they are the recipients of the version of elderspeak resulting from extended practice.

The young adults who participated in Experiment 1 did not report changing or altering their speech as a result of their five days of practice. Nor would it appear that they changed how they spoke to the older adults as a result of feedback from the task performance of their partners or as a result of their partners' verbalizations during the task. Task performance did not vary from session 1 to session 5, nor did the older adults' use of repetition, backchannel affirmatives, requests for clarification, or expressions of confusion. By session 5 , the young adults were overly familiar with the task; although new routes 
Kemper et al.: Practicing speech accommodations to older adults

were presented each day, the set of maps was repeated from session to session. Consequently, the young adults may not have been challenged by the task, and the experience of interacting with an older adult may have become routine. This would suggest that the speech accommodations seen at session 5 resulted from motivational and affective factors rather than cognitive ones.

This explanation, however, is somewhat weakened by the results of Experiment 2. Although the task surely had become equally familiar, routine, and repetitious for the young speakers, few practice effects were observed, and the fluency effects that were observed differed from those that occurred in Experiment 1 . Whereas extended practice with older partners resulted in a speech style characterized by shorter sentences, more fragments, and a slower rate of speech, extended practice with young partners resulted in a speech style characterized not only by shorter sentences and more fragments, but also by the use of fewer words and fewer utterances delivered at a faster rate. Although some caution is in order given the small sample size, it appears that extended practice with other. young adults does not affect prosody, grammatical complexity, semantic content, interaction style, or accuracy. It appears that extended practice with older partners forces the young adults to revise how they communicate; the changes in prosody, grammatical complexity, and semantic content observed in Experiment 1 as a result of the young adults' extended practice with older partners appear to arise from their cognitive assessment of the communication needs of their partners rather than simply from boredom and familiarity with the task.

This situation - routine, repetitive tasks and restricted opportunities for conversational interaction - is not unlike that experienced in nursing homes and other long-term care facilities. Nor was the version of elderspeak observed at session 5 much different from "secondary" babytalk, the form of elderspeak associated with such settings by Caporael (Caporael, 1981; Caporael \& Culbertson, 1986; Caporael, Lukaszewski, \& Culbertson, 1983). It may be that a highly simplified, restrictive, and patronizing form of elderspeak evolves as task interactions with older adults become routinized, nondemanding, and overly familiar. When younger adults first encounter older adults, they may adopt a simplified speech register that reduces processing demands and consequently enhances older adults' performance; with practice, this speech register may become so simplified that its use triggers negative self-evaluations by its recipients. Although this speech register may still enhance older adults' task performance, it may also decrease their self-assessments of communicative competence and, therefore, lead to increased social withdrawal and isolation.

\section{ACKNOWLEDGMENTS}

This research was supported by grants ROIAG09952 and KO4AG0043 from the National Institute on Aging.

\section{REFERENCES}

Ashburn, G., \& Gordon, A. (1981). Features of a simplified register in speech to elderly conversationalists. International Joumal of Psycholinguistics, 7, 31-43. 
Kemper et al.: Practicing speech accommodations to older adults

AVAAZ Innovations. (1995). Computerized Speech Research Environment 4.2 [computer software]. London, Ontario: Sound Scientific Solutions.

Caporael, L. (1981). The paralanguage of caregiving: Baby talk to the institutionalized aged. Journal of Personality and Social Psychology, 40, 876-884.

Caporael, L. R., \& Culbertson, G. H. (1986). Verbal response modes of baby talk and other speech at institutions for the aged. Language and Communication, 6, 99-112.

Caporael, L. R., Lukaszewski, M. P., \& Culbertson, G. H. (1983). Secondary babytalk: Judgments of institutionalized elderly and their caregivers. Journal of Personality and Social Psychology. 44, 746-754.

Chapman, R., \& Miller, J. (1984). SALT: Systematic Analysis of Language Transcripts. Madison: University of Wisconsin.

Edwards, H., \& Noller, P. (1993). Perceptions of overaccommodations used by nurses in communication with the elderly. Joumal of Language and Social Psychology, 12, 207-223.

Gibb, H., \& O'Brien, B. (1990). Jokes and reassurances are not enough: Ways in which nurses related through conversation with elderly clients. Joumal of Advanced Nursing, 15, 1389 1401.

Gubrium, J. F. (1975). Living and dying at Murray Manor. New York: St. Martin's.

Harwood, J., Giles, H., \& Ryan, E. B. (1995). Aging, communication, and intergroup therapy: Social identity and intergenerational communication. In J. Nussbaum \& J. Coupland (Eds.), Handbook of communication and aging (pp. 133-159). Hillsdale, NJ: Erlbaum.

Kemper, S. (1992). Language and aging. In F. I. M. Craik \& T. A. Salthouse (Eds.), Handbook of aging and cognition (pp. 213-270). Hillsdale, NJ: Erlbaum.

(1994). "Elderspeak": Speech accommodations to older adults. Aging and Cognition, 1, 1-10.

Kemper, S., Anagnopoulos, C., Lyons, K.. \& Heberlein, W. (1994). Speech accommodations to dementia. Joumal of Gerontology: Psychological Sciences, 49, P223-230.

Kemper, S., Othick, M., Warren, J., Gubarchuk, J., \& Gerhing, H. (1996). Facilitating older adults' performance on a referential communication task through speech accommodations. Aging. Neuropsychology, and Cognition, 3, 37-55.

Kemper, S., Vandeputte, D., Rice, K., Cheung, H., \& Gubarchuk, J. (1995). Speech adjustments to aging during a referential communication task. Journal of Language and Social Psychology, 14, 40-59.

Kintsch, W., \& Keenan, J. M. (1973). Reading rate and retention as a function of the number of the propositions in the base structure of sentences. Cognitive Psychology, 5, 257-274.

Lanceley, A. (1985). Use of controlling language in the rehabilitation of the elderly. Journal of Advanced Nursing, 10, 125-135.

Molfese, V. J., Hoffman, S., \& Yuen, R. (1981-1982). The influence of setting and task partner on the performance of adults over 65 on a communication task. International Joumal of Aging and Human Development, 14, 45-53.

O'Connor, B. P., \& Rigby, H. (1996). Perceptions of baby talk, frequency of receiving baby talk, and self-esteem among community and nursing home residents. Psychology and Aging, II, 147-154.

Rubin, K. H., \& Brown, I. D. R. (1975). A life-span look at person perception and its relationship to communicative interaction. Joumal of Gerontology, 30, 461-468.

Ryan, E. B., Bourhis, R. Y., \& Knops, U. (1991). Evaluative perceptions of patronizing speech addressed to elders. Psychology and Aging, 6, 442-450.

Ryan, E. B., Giles, H., Bartolucci, G., \& Henwood, K. (1986). Psycholinguistic and social psychological components of communication by and with the elderly. Language and Communication, 6, 1-24.

Ryan, E. B., Hamilton, J. M., \& Kwong See, S. (1994). Younger and older adult listeners' evaluations of baby talk addressed to institutionalized elders. International Journal of Aging and Human Development, 39, 21-32.

Ryan, E. B., Hummert, M. L., \& Boich, L. H. (1995). Communication predicaments of aging: Patronizing behavior toward older adults. Journal of Language and Social Psychology, 14, 144-166.

Ryan, E. B., Kwong See, S. K., Meneer, W. B., \& Trovato, D. (1992). Age-based perceptions of language performance among younger and older adults. Communication Research; 19, 423-443. 
Kemper et al.: Practicing speech accommodations to older adults

Ryan, E. B., MacLean, M., \& Orange, J. B. (1994). Inappropriate accommodation in communication to elders: Inferences about nonverbal correlates. International Joumal of Aging and Human Development, 39, 273-291.

Shipley, W. C. (1940). A self-administered scale for measuring intellectual impairment and deterioration. Joumal of Psychology, 9, 371-377.

Warren, A., \& McCloskey, L. A. (1997). Language in social contexts. In J. Berko Gleason (Ed.), The development of language (4th ed., pp. 210-258). Boston: Allyn \& Bacon. 